\title{
Out-of-equilibrium dynamics of repulsive Fermi gases in quasiperiodic potentials: A density functional theory study
}

\author{
Francesco Ancilotto, ${ }^{1,2}$ Davide Rossini, ${ }^{3}$ and Sebastiano Pilati ${ }^{1,4}$ \\ ${ }^{1}$ Dipartimento di Fisica e Astronomia “Galileo Galilei”, Università di Padova, via Marzolo 8, Padova 35122, Italy \\ ${ }^{2}$ CNR-IOM Democritos, via Bonomea, Trieste 265-34136, Italy \\ ${ }^{3}$ Dipartimento di Fisica, Università di Pisa and INFN, Largo Pontecorvo 3, Pisa I-56127, Italy \\ ${ }^{4}$ School of Science and Technology, Physics Division, University of Camerino, Via Madonna delle Carceri 9, Camerino I-62032, Italy
}

(Received 20 January 2018; published 5 April 2018)

\begin{abstract}
The dynamics of a one-dimensional two-component Fermi gas in the presence of a quasiperiodic optical lattice (OL) is investigated by means of a density functional theory approach. Inspired by the protocol implemented in recent cold-atom experiments - designed to identify the many-body localization transition-we analyze the relaxation of an initially prepared imbalance between the occupation number of odd and of even sites. For quasidisorder strength beyond the Anderson localization transition, the imbalance survives for long times, indicating the inability of the system to reach local equilibrium. The late-time value of the imbalance diminishes for increasing interaction strength. Close to the critical quasidisorder strength corresponding to the noninteracting (Anderson) transition, the interacting system displays an extremely slow relaxation dynamics, consistent with subdiffusive behavior. The amplitude of the imbalance fluctuations around its running average is found to decrease with time, and such damping is more effective with increasing interaction strengths. While our study addresses the setup with two equally intense OLs, very similar effects due to interactions have been observed also in recent cold-atom experiments performed in the tight-binding regime, i.e., where one of the two OLs is very deep and the other is much weaker.
\end{abstract}

DOI: 10.1103/PhysRevB.97.155107

\section{INTRODUCTION}

Since Anderson's 1958 seminal paper [1], it is known that sufficiently strong disorder can cause the localization of noninteracting quantum particles, inducing an insulating behavior in macroscopic samples. A vast body of more recent theoretical work supports the view that localization can persist also in the presence of interactions [2], leading to a nonergodic phase of matter - dubbed many-body localized (MBL) phase-which fails to thermalize, thus violating the eigenstate thermalization hypothesis (for a review, see Ref. [3]). However, the MBL phase is believed to be qualitatively different from the noninteracting Anderson insulator: while both phases are characterized by the absence of transport of any physical quantity, quantum correlations can still propagate in the MBL phase. The latter observation comes from the possibility to map the MBL system to an integrable one, with an extensive number of localized constants of motion [4,5]: even if local observables reach stationary, and nonthermal, values, the coherences of far apart sites evolve nontrivially in time, thus resulting in a slow persistent dephasing [6]. The logarithmic growth in time of the bipartite entanglement entropy [7-9] or the power-law decay of two-site entanglement $[10,11]$ are two distinctive signatures of that mechanism, to be contrasted with a saturating behavior in the Anderson localized phase.

In the last few years cold-atom setups have been employed to experimentally investigate localization phenomena in disordered and interacting quantum systems, exploiting the direct control of interactions and disorder that these systems allow [12-15]. In particular, in a series of experiments reported in Refs. [16-18], the dynamics of a one-dimensional (1D) atomic Fermi gas exposed to a quasiperiodic potential has been explored. The protocol consisted in preparing an initial density modulation, and in studying the ensuing relaxation dynamics for different degrees of (quasi)disorder and interaction strengths. The long-time persistence of the initial density imbalance, which signals the failure of a local observable to equilibrate, was interpreted as a signature of MBL.

The computational studies of this kind of dynamics usually employ very accurate, but also extremely demanding, exact diagonalization or density-matrix renormalization group (DMRG) calculations [19], which are limited to small-sized, discrete lattice models and short evolution times. Such limitations are of particular relevance in the ergodic phase, where the bipartite entanglement entropy spreads linearly in time, thus making long-time DMRG calculations unfeasible.

In this work we use time-dependent density functional theory (TD-DFT) in order to simulate the dynamics of a pseudodisordered two-component 1D Fermi gas with contact repulsive interactions. The gas is subject to the quasiperiodic potential generated by two optical lattices (OLs) with incommensurate periods, but equal intensities (this intensity plays the role of quasidisorder strength). Specifically, we analyze the evolution of an initially prepared configuration with a density modulation such that the odd sites are empty and the even sites are doubly occupied. The exchange-correlation functional, which is the main ingredient of the TD-DFT formalism and embodies correlation effects beyond the Hartree mean-field description, is derived using an adiabatic local spin-density approximation (LSDA), based on the exact Bethe-ansatz solution for the homogeneous system [20-22]. 
The accuracy of this DFT method in predicting ground-state properties has been carefully tested in a recent work by making extensive comparisons against unbiased quantum Monte Carlo simulations [23], showing that the LSDA approach is very reliable in a broad range of interaction strengths and of OL intensities. Furthermore, the accuracy of the dynamics obtained within the adiabatic approximation for the exchange and correlation functional has been verified in the context of the 1D Hubbard model by making quantitative comparison against essentially exact time-dependent DMRG calculations [24]. We thus argue that the TD-DFT method we employ represents a useful complement with respect to more accurate but more demanding methods such as DMRG calculations, allowing us to address larger system sizes and longer evolution times. We remark that the TD-DFT theory in the present formulation is not restricted to small deviations from the ground-state density, but can be reliable well outside the linear-response regime [25].

Furthermore, the DFT approach is based on a continuousspace model, as opposed to the discrete-lattice tight-biding approximations usually addressed by exact-diagonalization and DMRG calculations. This allows us to consider the experimental setup with two equally intense OLs, which is characterized by the presence of mobility edges separating localized from extended single-particle states [26-29], in contrast to the tight-binding Aubry-André model [30]—which approximately describes the setup where one OL is very deep and the other is much weaker-for which all eigenstates localize at the same quasidisorder strength. The important effects due to the presence of single-particle mobility edges [31] beyond the strict tight-binding regime have indeed been observed and emphasized in a recent experiment [32].

In the noninteracting case, our calculations show that the density imbalance between odd and even sites rapidly vanishes for weak quasidisorder, while it survives in the long-time limit for quasidisorder strengths beyond the critical point of the Anderson localization. In the interacting case, the long-time value of the imbalance at strong quasidisorder is substantially reduced compared to the noninteracting case, indicating that interactions have a delocalizing effect. Interestingly, when we tune the quasidisorder strength close to and slightly above the noninteracting Anderson transition, the interacting system displays an extremely slow relaxation dynamics, consistent with a dynamical critical exponent larger than $z=2$, thus indicating subdiffusive behavior. This phenomenon has been recently observed in experiments [17] performed in the tightbinding regime, and it has been interpreted as a Griffiths effect. The temporal fluctuations of the imbalance around its running average are found to decay in time and the damping of the fluctuation amplitude is found to be more effective with increasing interaction strengths. These effects have been observed in previous DMRG simulations of the Aubry-André model, and have been attributed to the growth of the entanglement entropy [17].

The remainder of the paper is organized as follows: in Sec. II we provide the details of the model we simulate and we describe the TD-DFT computational method used here. The results for the relaxation dynamics of the initially imprinted density wave are reported in Sec. III. In Sec. IV we draw our conclusions.

\section{METHODS}

The 1D atomic Fermi gas considered in this paper is described by the following continuous-space Hamiltonian:

$$
\hat{H}=\sum_{i=1}^{N}\left(-\frac{\hbar^{2}}{2 m} \frac{d^{2}}{d x_{i}^{2}}+v\left(x_{i}\right)\right)+\sum_{i_{\uparrow}, i_{\downarrow}} g \delta\left(x_{i \uparrow}-x_{i \downarrow}\right) .
$$

Here $N=N_{\uparrow}+N_{\downarrow}$, where $N_{\uparrow}, N_{\downarrow}$ are the numbers of atoms in the two fermionic components, hereafter referred to as spin-up and spin-down particles. The coupling constant $g$ is related to the $1 \mathrm{D}$ scattering length $a_{1 \mathrm{D}}, g=-2 \hbar^{2} /\left(m a_{1 \mathrm{D}}\right)$ (with $\hbar$ the reduced Planck constant and $m$ the atomic mass). We consider here purely repulsive interactions, i.e., $g \geqslant 0$.

The Hamiltonian (1) faithfully describes cold-atom experiments performed in tight cigar-shaped traps, and the value of $a_{1 \mathrm{D}}$ can be determined from the (three-dimensional) $s$-wave scattering length and the radial confining strength [33]. It is convenient to introduce the adimensional interaction parameter $\gamma=m g /\left(\hbar^{2} n\right)=2 /\left(n\left|a_{1 \mathrm{D}}\right|\right)$ where $n=N / L$ is the total density and $L$ is the system size. The $\gamma \rightarrow 0\left(a_{1 \mathrm{D}} \rightarrow-\infty\right)$ limit corresponds to a noninteracting Fermi gas, while the $\gamma \rightarrow \infty$ $\left(a_{1 \mathrm{D}} \rightarrow 0^{-}\right)$limit corresponds to a strongly interacting regime, where distinguishable fermions fermionize [34,35], i.e., their energy and density can be mapped to those of indistinguishable (spin polarized) fermions [36,37]. A 1D (quasi)disorder can be introduced by superimposing two OLs with incommensurate periods, one with a (short) period $d_{s}$, and another with a longer period $d_{l}$, thus resulting in an external potential of the form $v(x)=V_{0}\left[\sin ^{2}\left(\pi x / d_{s}\right)+\sin ^{2}\left(\pi x / d_{l}\right)\right]$. The OL intensity $V_{0}$ plays the role of quasidisorder strength. Hereafter $V_{0}$ will be conveniently expressed in units of the recoil energy $E_{r}=$ $\hbar^{2} \pi^{2} /\left(2 m d_{s}^{2}\right)$ of the short-period lattice.

In order to simulate an infinite quasiperiodic potential, the ratio $d_{l} / d_{s}$ between the two periods must be an irrational number. We choose the golden ratio $\phi=(\sqrt{5}+1) / 2$ for such a number. Our simulations address a finite box with periodic boundary conditions, since generally they reduce finite-size effects compared to, e.g., open boundary conditions. To make the potential $v(x)$ consistent with the use of periodic boundary conditions, one needs to approximate this number by the ratio of two integer numbers, the largest one providing the total length of the periodic cell used in the calculation. Here we approximate $\phi$ with the ratio of two successive numbers in the Fibonacci sequence: $d_{l} / d_{s}=F_{k+1} / F_{k}$ [38], which converges towards the golden ratio for large values of $k$. The potential $v(x)$ thus complies with periodic boundary conditions, still being aperiodic within the simulated cell of length $L=F_{k+1} d_{s}$. In the following we set (unless otherwise stated) $F_{k}=89$ and $F_{k+1}=$ 144 , corresponding to a total OL length $L / d_{s}=144$. We focus on a half-filled lattice, with $N_{\uparrow}=N_{\downarrow}=72$ particles (unless otherwise specified), so that on average there is one fermion per well of the short-period lattice. It has been recently shown that, in a single half-filled OL, interparticle interactions play an important role, causing the formation of quasi-long-range antiferromagnetic order [39]. It is also worth emphasizing that a system comprising $N=144$ fermions cannot be addressed via exact diagonalization calculations (see, e.g., the Krylov subspace technique of Ref. [40]), and is out of reach also for any time-dependent DMRG simulation, except perhaps in 
the strongly localized regime, where the entanglement entropy does not rapidly grow.

We choose to simulate the dynamics of the Hamiltonian (1) by employing a TD-DFT approach. DFT has recently entered the field of ultracold gases as a useful computational tool that goes beyond the usual mean-field approximation, which is often used to model such systems. Recent applications of DFT methods to ultracold fermionic systems allowed us to study the ferromagnetism and antiferromagnetism in repulsive Fermi gases in shallow OLs [41], vortex dynamics in superfluid Fermi gases $[42,43]$, superfluidity and density modulations in dipolar Fermi gases [44], vortices in rotating dipolar Fermi gases [45], and the formation of ferromagnetic domains in trapped clouds [46]. DFT has also been used to study strongly correlated Fermi gases in elongated harmonic traps [20].

In a recent paper [23], the accuracy of the LSDA for 1D repulsive Fermi gases in OLs has been assessed. To this aim, quantum Monte Carlo (QMC) simulations based on the fixed-node method to circumvent the sign problem were employed, providing exact results for the 1D system of interacting fermions [47]. A systematic comparison between DFT calculations of ground-state energies and density profiles for a half-filled OL against the outcomes of the QMC simulations allowed the authors of Ref. [23] to determine a wide range of OL intensities and interaction strengths where the LSDA appears to provide quite accurate predictions. The accuracy of DFT (in the LSDA) in 1D fermionic systems has been also demonstrated for small finite systems in Ref. [48]. We note at this point that a possible improvement over LSDA for systems characterized by strongly spatially localized states (like those arising, e.g., from confinement within deep optical lattices) could be the addition of gradient corrections, very much like what is currently done in electronic structure calculations using gradient-corrected exchange-correlation density functionals [49]. However, including these corrections does not seem to be necessary here: the comparisons made in Ref. [23] showed that, for strengths of the optical lattices similar to the ones considered here, the LSDA approach already gives results in excellent agreement with unbiased QMC calculations.

To study the real-time dynamics of the system, we use here the so-called "adiabatic" LSD approximation, where the time-dependent exchange-correlation (xc) potential is represented by the static xc potential (treated within the LSD approximation) evaluated at the instantaneous density. The theory is thus local in time, as well as in space ("memory" effects are ignored). An appealing feature of this theory is that it satisfies Galilean invariance [50]. The adiabatic TD-DFT approach (in the LSDA) to inhomogeneous fermion systems in one dimension has been extensively tested in Ref. [24] and its accuracy in describing collective density and spin dynamics in strongly correlated 1D ultracold Fermi gases has been proved by comparing TD-DFT predictions with accurate results based on DMRG calculations, finding remarkable agreement even in a regime of large and rapid deviations from the initial ground-state densities, where the linear-response theory is hardly applicable. Notice also that this scheme has been shown to be suitable to account for the dynamics of spin-charge separation, a remarkable phenomenon of 1D Fermi systems, although a nonadiabatic correction term was needed to account for spin-drag effects [51].
Including current terms or effective mass corrections might, in principle, increase the accuracy of the adiabatic TD-DFT approach used here. These corrections have been employed, e.g, in studies of the dynamics of three-dimensional attractive superfluid Fermi gases [52]. However, we will not further explore these possibilities here.

The Kohn-Sham (KS) formulation [53] of DFT [50] for an inhomogeneous system of $N$ interacting particles with spin projection $\sigma=\uparrow, \downarrow$ is based on the following energy functional of the density:

$$
E_{\mathrm{KS}}\left[n_{\uparrow}, n_{\downarrow}\right]=\frac{\hbar^{2}}{2 m} \sum_{\sigma} \sum_{i=1}^{N_{\sigma}} \int\left|\nabla \phi_{i}^{\sigma}(x)\right|^{2} d x+E_{\mathrm{HXC}}\left[n_{\uparrow}, n_{\downarrow}\right] .
$$

The $\left\{\phi_{i}^{\sigma}(x)\right\}_{i=1, \ldots, N_{\sigma}}$ are single-particle orbitals forming orthonormal sets, $\left\langle\phi_{i}^{\sigma} \mid \phi_{j}^{\sigma}\right\rangle=\delta_{i j}$, filled up to the Fermi level. The spin-resolved density is given by $n_{\sigma}(x)=\sum_{i=1}^{N_{\sigma}}\left|\phi_{i}^{\sigma}(x)\right|^{2}$, so that the total density of the system is $n(x)=n_{\uparrow}(x)+$ $n_{\downarrow}(x)$. The interaction energy functional $E_{\mathrm{HXC}}$, which includes the mean-field (Hartree) energy and the exchange-correlation contribution, is treated here within the LSDA, i.e.,

$$
E_{\mathrm{HXC}}=\int d x n(x) \epsilon_{\mathrm{HXC}}^{\mathrm{hom}}\left(n_{\uparrow}(x), n_{\downarrow}(x)\right),
$$

where $\epsilon_{\mathrm{HXC}}^{\mathrm{hom}}$ is the corresponding energy per particle in the homogeneous phase. The latter can be written using the exact Bethe-ansatz solution for the ground-state energy as

$$
\epsilon_{\mathrm{HXC}}^{\mathrm{hom}}=\frac{1}{N}\left(E_{\mathrm{tot}}^{\mathrm{hom}}-E_{\mathrm{kin}}^{\mathrm{hom}}\right)=\frac{\hbar^{2}}{2 m} n^{2} f(\gamma, P),
$$

where

$$
\frac{E_{\text {kin }}^{\text {hom }}}{N}=\frac{\pi^{2} \hbar^{2} n^{2}}{24 m}\left(1+3 P^{2}\right)
$$

is the kinetic energy of the homogeneous noninteracting system, and $f(\gamma, P)=\left(\pi^{2} / 4\right) f_{\text {exa }}(\gamma, P)$. Here $P(x)=\left[n_{\uparrow}(x)-\right.$ $\left.n_{\downarrow}(x)\right] / n(x)$ denotes the local polarization. The term $f_{\text {exa }}$ is given by [21]

$$
\begin{aligned}
f_{\text {exa }}= & {[\eta(x)-1 / 3]\left\{1+\alpha(x) P^{2}+\beta(x) P^{4}\right.} \\
& \left.-[1+\alpha(x)+\beta(x)] P^{6}\right\},
\end{aligned}
$$

where $x \equiv 2 \gamma / \pi$ and

$$
\begin{aligned}
& \alpha(x)=\frac{\left(-x^{2}+a_{\alpha} x+b_{\alpha}\right)}{x^{2}+c_{\alpha} x-b_{\alpha}}, \\
& \beta(x)=\frac{a_{\beta} x}{x^{2}+b_{\beta} x+c_{\beta}}, \\
& \eta(x)=\frac{4 x^{2} / 3+a_{P} x+b_{P}}{x^{2}+c_{P} x+d_{P}} .
\end{aligned}
$$

Here $a_{\alpha}=-1.68894, b_{\alpha}=-8.0155, c_{\alpha}=2.74347, a_{\beta}=$ $-1.51457, b_{\beta}=2.59864, c_{\beta}=6.58046, a_{P}=5.780126$, $b_{P}=-(8 / 9) \ln 2+\pi a_{P} / 4, c_{P}=(8 / \pi) \ln 2+3 a_{P} / 4, d_{p}=$ $3 b_{P}$.

Constrained minimization of the functional $E_{\mathrm{KS}}$ leads to the coupled KS eigenvalues equations:

$$
\hat{H}_{\mathrm{KS}} \phi_{i}^{\sigma}(x) \equiv\left[-\frac{\hbar^{2}}{2 m} \frac{d^{2}}{d x^{2}}+v(x)+V_{\sigma}(x)\right] \phi_{i}^{\sigma}(x)=\epsilon_{i} \phi_{i}^{\sigma}(x) .
$$


The effective potential $V_{\sigma}(x) \equiv \delta E_{\mathrm{HXC}} / \delta n_{\sigma}(x)=\partial\left(n \epsilon_{\mathrm{HXC}}\right) /$ $\partial n_{\sigma}$ can be written as

$$
\begin{aligned}
V_{\sigma} & =\frac{\hbar^{2}}{2 m}\left[f(\gamma, P) \frac{\partial n^{3}}{\partial n_{\sigma}}+n^{3} \frac{\partial f}{\partial n_{\sigma}}\right] \\
& =\frac{\hbar^{2}}{2 m}\left[3 n^{2} f-n^{2} \gamma \frac{\partial f}{\partial \gamma} \pm 2 n n_{-\sigma} \frac{\partial f}{\partial|P|} \frac{P}{|P|}\right],
\end{aligned}
$$

where we used the fact that $\partial n / \partial n_{\sigma}=1, \partial P / \partial n_{\sigma}= \pm 2 n_{-\sigma} / n^{2}$, and $\partial \gamma / \partial n=-\gamma / n$. Therefore $V_{\sigma}$ couples only fermions with opposite polarization, since we consider a zero range model for the interatomic interaction.

In the following we seek time-dependent solutions $\left\{\phi_{i}^{\sigma}(x, t)\right\}_{i=1, \ldots, N_{\sigma}}$ by propagating in real time the timedependent version [53] of the KS equations (10), i.e., $i \hbar \partial \phi_{i}^{\sigma} / \partial t=\hat{H}_{\mathrm{KS}} \phi_{i}^{\sigma}$. Both the densities $n_{\sigma}(x)$ and the orbitals $\phi_{i}^{\sigma}(x)$ are discretized in Cartesian coordinates using a spatial grid fine enough to guarantee well converged values of the total energy $E_{\mathrm{KS}}$. The orthogonality between different orbitals is enforced by a Gram-Schmidt process. The spatial derivative entering Eq. (10) is calculated with accurate 13-point formulas. The time-dependent Schrödinger's equation (10) is solved using an Hamming's predictor-modifier-corrector method [54], initiated by a fourth-order Runge-Kutta-Gill algorithm $[54,55]$. This choice provides excellent stability and energy conservation even during simulations spanning rather long-time intervals.

\section{RESULTS}

In order to discern the delocalized ergodic phase from the insulating (putative MBL) phase, we follow a protocol similar to the one used in a series of recent experiments [16-18,32]. We create an initial state with a density modulation, such that the even sites of the short-period OL are almost empty and the odd sites are almost doubly occupied. This is achieved by computing the ground state of the Hamiltonian (1) in the presence of an additional superimposed OL with period $2 d_{s}$ and a well depth which is twice the chosen value of $V_{0}$ [56].

The dynamics of this initial state is determined via the TDDFT method described in Sec. II. In particular, we compute the time-dependent imbalance $\mathcal{I}(t)$ between the respective atom number on even $N_{e}$ and odd $N_{o}$ sites:

$$
\mathcal{I}(t)=\frac{N_{e}-N_{o}}{N_{e}+N_{o}} .
$$

In the noninteracting case, the imbalance $\mathcal{I}$ rapidly reaches negligibly small values for quasidisorder strengths smaller than $V_{0} \simeq 1.06 E_{r}$, as shown in Fig. 1, indicating that the system is indeed able to equilibrate. For higher values of the quasidisorder strength $V_{0}, \mathcal{I}$ remains finite in the long-time limit. Its asymptotic value $\langle\mathcal{I}\rangle$ (computed as described below) increases with $V_{0}$ for disorder strengths above the critical point.

The position of the calculated critical point for the noninteracting system is consistent with the quasidisorder strength necessary to induce Anderson localization of the single-particle eigenstates in the low-energy regime of the spectrum, equal to $V_{0} \simeq 1.1 E_{r}$. We determine this value by analyzing the scaling with system size of the average of the participation ratios (which is a measure of the spatial extent of a single-particle

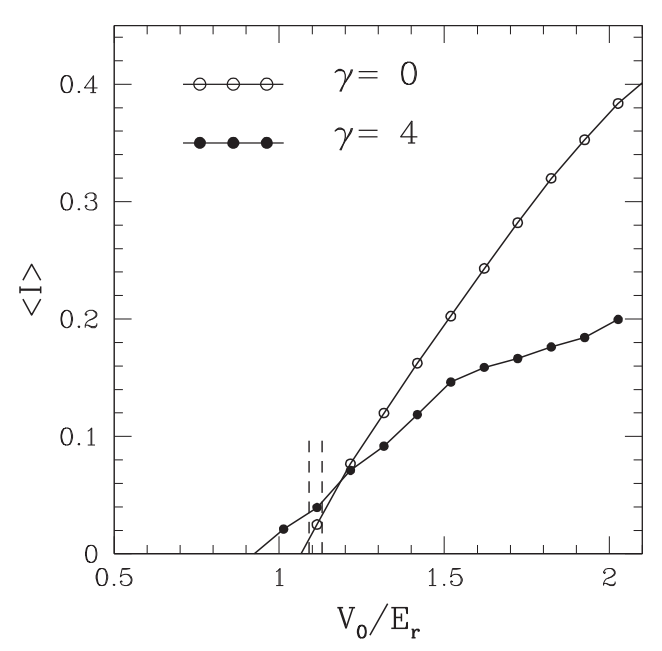

FIG. 1. Late-time value of the imbalance $\langle\mathcal{I}\rangle$ as a function of the quasidisorder strength $V_{0} / E_{r}$, computed as the average of $\mathcal{I}(t)$ within the time window $t \in[80 \tau: 100 \tau]$ after the relaxation starts. We choose $\tau=2 m d_{s}^{2} / \hbar$ as the time unit. Two cases are shown: the noninteracting $(\gamma=0)$ and the interacting $(\gamma=4)$ case. In the former case, the dynamical evolution of $\mathcal{I}(t)$ rapidly saturates, so that the values of $\langle\mathcal{I}\rangle$ shown here represent the asymptotic stationary value. In the latter case, $\mathcal{I}(t)$ undergoes an extremely slow drift if $V_{0} / E_{r} \gtrsim 1.1$ (see Fig. 3), making it unfeasible to identify an asymptotic stationary value within the achievable simulation times. The vertical dashed segments bracket the critical point of the noninteracting Anderson localization transition, computed by analyzing the participation ratios of the low-energy single-particle orbitals (see text).

wave function [57]) of the lowest $L /\left(60 d_{s}\right)$ eigenstates (the vertical segments shown in Fig. 1 bracket the so-determined critical point). This suggests that, as soon as some of the singleparticle eigenstates are spatially localized, the asymptotic value of $\langle\mathcal{I}\rangle$ is finite. It is worth emphasizing that, as opposed to the Aubry-André model - for which all eigenstates localize at the same quasidisorder strength - in the continuous-space model of Eq. (1) the critical quasidisorder strength depends on the energy of the state $[26-28,39]$. In particular, the low-energy eigenstates localize at weaker quasidisorder strength compared to high-energy states.

Introducing interactions among fermions (i.e., $\gamma>0$ ) causes important effects. For strong quasidisorder, the latetime value of $\mathcal{I}$ is significantly reduced compared to the noninteracting case, while for intermediate quasidisorder strengths this reduction is less pronounced. This is apparent in Fig. 2, where we plot the late-time average value $\langle\mathcal{I}\rangle$ versus interaction strength $\gamma$ for different quasidisorder intensities $V_{0}$. The values of $\langle\mathcal{I}\rangle$ displayed in Figs. 1 and 2 are computed by averaging the calculated values $\mathcal{I}(t)$ over the last portion, $\sim 20 \tau$, of a total simulation time $t_{\max } \sim 100 \tau$. Here $\tau \equiv 2 m d_{s}^{2} / \hbar$ is the unit of time. A word of caution is in order here. The time evolutions of the imbalance $\mathcal{I}$ in the noninteracting case and in the interacting case are qualitatively different. While in the former case $\mathcal{I}(t)$ rapidly saturates to the asymptotic value, and then undergoes virtually random fluctuations around the mean value, in the latter case, if the quasidisorder strength is close to or slightly beyond the critical point of the (noninteracting) Anderson transition, we observe an extremely slow drift 


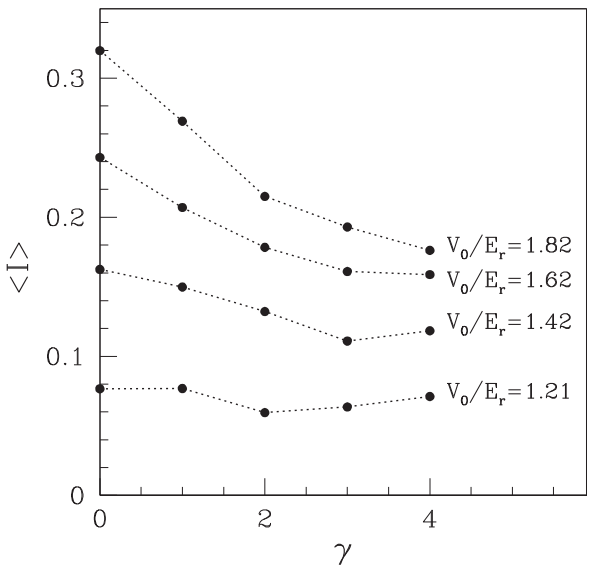

FIG. 2. Late-time average value of the imbalance $\langle\mathcal{I}\rangle$, for various values of the quasidisorder strength $V_{0} / E_{r}$, shown as a function of the interaction strength $\gamma$.

towards lower values, i.e., longer relaxation times. Therefore, in the interacting case the late-time average imbalance $\langle\mathcal{I}\rangle$ measured at quasidisorder strengths close to the Anderson transition should not be interpreted as an asymptotic stationary value, but rather as a transient value observed at an intermediate time along an extremely slow relaxation dynamics. This slow relaxation is illustrated in Fig. 3, where we show the different dynamics associated with various interaction strengths. A similar slowdown of the dynamics has previously been observed in recent experiments [17], and also in exact-diagonalization calculations [17] (see also Refs. [58,59]), both performed in the tight-binding regime, and it was interpreted as a consequence of the Griffiths effect. This effect is characteristic of purely random systems, where statistical spatial variations of the external random field create subregions with stronger disorder, which have a local insulating character. While such subregions

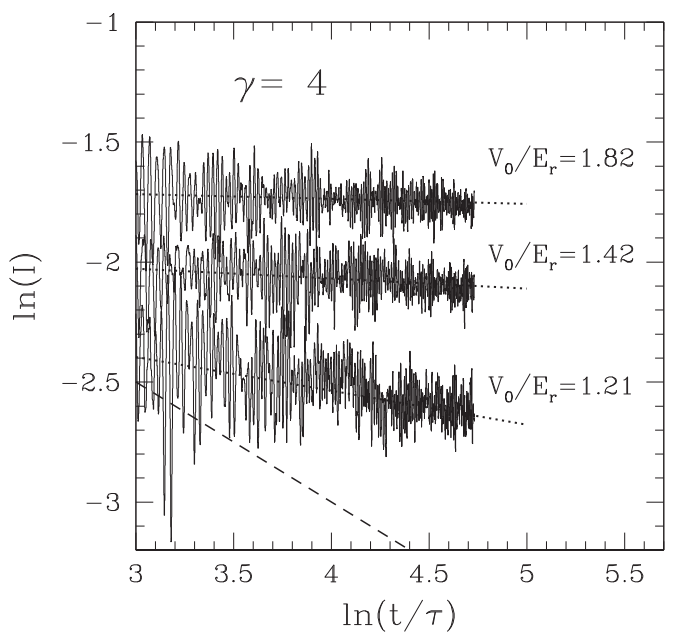

FIG. 3. Imbalance $\mathcal{I}$ as a function of evolution time $t$ for the interacting gas at $\gamma=4$, in three bichromatic OLs with intensities (from bottom to top) $V_{0} / E_{r}=1.21,1.42,1.82$. The dotted lines represent power-law fits of the type $\mathcal{I} \sim t^{-1 / z}$, where $z$ is the dynamical critical exponent. The dashed line corresponds to $z=2$, which characterizes a purely diffusive behavior.

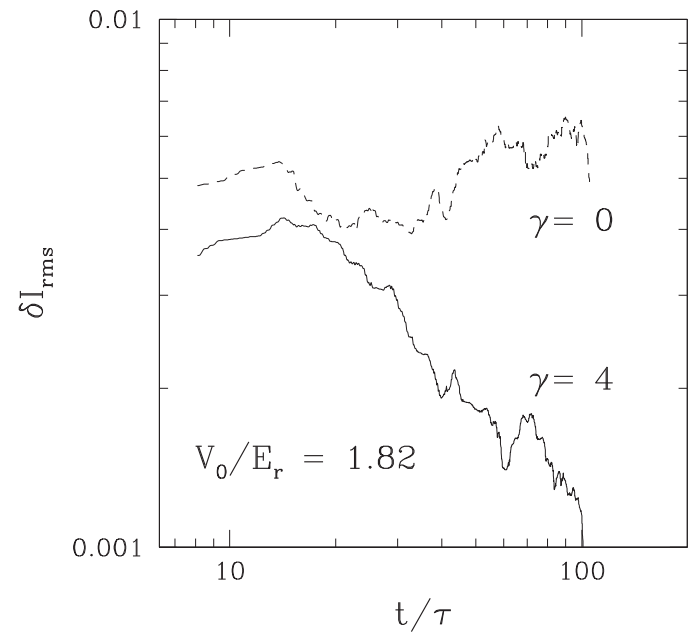

FIG. 4. Average fluctuation of the imbalance around its running average, as a function of time, for two values of the interaction strength $\gamma$. The quasidisorder strength is $V_{0} / E_{r}=1.82$.

will eventually thermalize with the surrounding (thermal) regions, they cause a slowdown of the overall dynamics. The existence of the Griffiths phenomenon for quasiperiodic systems has been challenged [60], due to the absence of purely random statistical fluctuations. In Ref. [17], the occurrence of Griffiths effects in the quasiperiodic system was attributed to the randomness of the initial state, in which the spin distribution was disordered, causing a different local impact of the interactions. It is indeed remarkable that a similar effect is observed also in our study, where the initial state is instead ordered (i.e., an alternation of almost empty and doubly occupied sites).

Following the theoretical analysis of Ref. [17], we fit the decay with time of the imbalance with the power law $\mathcal{I} \sim t^{-1 / z}$, where the dynamical critical exponent $z$ associated to transport is used as a fitting parameter. In the quasidisorder range $1.1 \lesssim$ $V_{0} / E_{r} \lesssim 1.4$ we obtain values larger than $z=2$ (which would correspond to diffusive dynamics), thus indicating subdiffusive behavior. For larger quasidisorder strength, the imbalance (after an initial rapid decay) remains essentially constant for the observable timescale, consistently with the emergence of a (putative MBL) phase which fails to equilibrate. It is worth emphasizing that the total timescale of our simulations is comparable to the longest evolution times achieved in the recent cold-atom experiments, and it is two orders of magnitude longer than the microscopic timescale for singleparticle tunneling between nearest-neighbor wells of a single OL with intensity $V_{0} \sim E_{r}$. The recent cold-atom experiments employed deeper OLs, where the tunneling time is somewhat longer than the one corresponding to our setup, so that the total observable timescale was approximately 40 times longer than the tunneling time. It is remarkable that memory of the initial configuration survives for times so much longer than the single-particle tunneling time.

Interactions have a relevant impact also on the temporal fluctuations of the imbalance. In order to elucidate this effect, we characterize the amplitude of these fluctuations using the root-mean-squared deviation $\delta \mathcal{I}_{\text {rms }}$ around the running average 


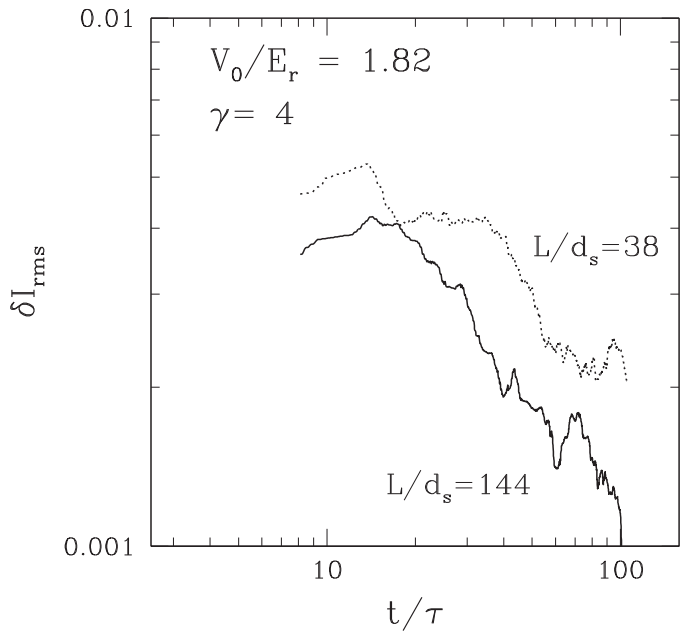

FIG. 5. Average fluctuation of the imbalance around its running average, as a function of time, for the case $V_{0} / E_{r}=1.82$ and two system sizes $L / d_{s}$. Dashed line: $L / d_{s}=38$; solid line: $L / d_{s}=144$.

$\langle\mathcal{I}\rangle_{\text {run }}$, evaluated within a temporal window of width $\Delta t \sim$ $t_{\max } / 7$. Here

$$
\delta \mathcal{I}_{\text {rms }} \equiv \sqrt{\frac{1}{N_{s}} \sum_{j=1}^{N_{s}}\left(\mathcal{I}\left(t_{j}\right)-\langle\mathcal{I}\rangle_{\text {run }}\right)^{2}}
$$

where the sum and the running average $\langle\ldots\rangle_{\text {run }}$ are performed over $N_{s}$ time steps $t_{j}\left(j=1, \ldots, N_{s}\right)$ within the temporal window. When the interaction strength increases, the asymptotic value of $\delta \mathcal{I}_{\text {rms }}$ diminishes substantially compared to the noninteracting case, where the imbalance fluctuations appear instead to undergo virtually random oscillations, after an initial decay. This is illustrated in Fig. 4. This interaction effect has been discussed previously in Ref. [16] based on timedependent DMRG simulations of the Aubry-André model. It was also found that the rate of suppression of $\delta \mathcal{I}_{\text {rms }}$ with time is related to the growth rate of the entanglement entropy, suggesting that measuring the fluctuations of $\mathcal{I}$ might allow one to extract information about the entanglement entropy-a nonlocal quantity - from a local observable.

It is worth noticing that the finite oscillation amplitude $\delta \mathcal{I}_{\text {rms }}$ we measure in the long-time limit in the interacting system might be due to the finite system size. Indeed, as shown in Fig. 5, the asymptotic value is significantly smaller for larger system sizes.

\section{CONCLUSIONS}

We have studied the dynamics of a two-component 1D Fermi gas with contact repulsive interactions, and subject to a quasiperiodic potential formed by two OLs with incommensurate periods. The setup we considered, in which the two OLs have the same intensity, has been addressed before via equilibrium ground-state QMC simulations, which allow us to discern the metal-insulator transition at zero temperature [29]. In this paper we extended the previous study by addressing the out-of-equilibrium dynamics via the TD-DFT method, following a protocol similar to the one implemented in a series of recent cold-atom experiments [16-18,32] aimed at investigating the MBL phenomenon. This protocol consists in following the relaxation of an initially imprinted density imbalance, and allowed the experimentalists to identify a nonergodic phase where the initially imprinted density imbalance survives after long times, thus signaling the inability of the system to reach local thermal equilibrium. This is one of the features characterizing MBL phases [3].

Our simulations displayed several of the most relevant phenomena observed in the experiments, which however have been performed in the tight-binding setup, where one of the two OLs is very deep and the other is much weaker. Among other effects, we observed a sizable reduction of the longtime value of the imbalance-which is finite in the strong quasidisorder regime - due to weak and intermediate repulsive interactions. These results represent a quantitative benchmark which might be useful for future experiments performed beyond the tight-binding regime. Furthermore, we observed an extreme slowdown of the dynamics of the interacting system in the vicinity of the noninteracting (Anderson) transition, and also a decrease in time of the imbalance fluctuations. This decrease is quite pronounced in the interacting system, while it is essentially negligible in the noninteracting case. We underline that the continuous-space model we consider here differs substantially from the Aubry-André model (which approximates the experimental system in the tight-binding regime). For this reason we do not observe the reentrant behavior observed in the experiment, where the late-time value of the imbalance was found to increase in the strongly interacting limit. This reentrance is due the fact that, in this limit, the dynamics can be described using a noninteracting fermion model [16]. While in the Aubry-André model all single-particle states-which determine the dynamics of the noninteracting model-localize at the same quasidisorder strength, in the continuous-space model (1) high-energy extended states are present also at strong quasi-disorder. Furthermore, in the latter model atoms in doubly occupied sites (referred to as doublons in Ref. [16]) can separate also in the strongly interacting limit, while in the Aubry-André model they become, in this limit, stable quasiparticles which tunnel only with an effective second-order tunneling, henceforth favoring localization.

The TD-DFT method implemented here represents a useful complement to more accurate but more demanding techniques such as, e.g., DMRG calculations. Indeed, it allows us to address larger systems sizes and longer evolution times, and also to simulate realistic continuous-space models as opposed to tight-binding approximations. Furthermore, TD-DFT can be extended to higher dimensions at an affordable computational price. In contrast, DMRG has revealed a powerful method to address the ground state of ladder and even 2D systems (being unbiased with respect to any sign problem) [61], but its cost remains exponential in the system width, and its extension to time-dependent calculations is problematic, and it is still the subject of ongoing research in the tensor-network community.

\section{ACKNOWLEDGMENTS}

We acknowledge a CINECA award under the ISCRA initiative, for the availability of high performance computing 
resources and support. S.P. and F.A. acknowledge financial support from the BIRD 2016 project "Superfluid properties of
Fermi gases in optical potentials" of the University of Padova. Fruitful discussions with R. Fazio are acknowledged.
[1] P. W. Anderson, Phys. Rev. 109, 1492 (1958).

[2] D. Basko, I. Aleiner, and B. Altshuler, Ann. Phys. 321, 1126 (2006).

[3] R. Nandkishore and D. A. Huse, Annu. Rev. Condens. Matter Phys. 6, 15 (2015).

[4] M. Serbyn, Z. Papić, and D. A. Abanin, Phys. Rev. Lett. 111, 127201 (2013).

[5] D. A. Huse, R. Nandkishore, and V. Oganesyan, Phys. Rev. B 90, 174202 (2014).

[6] M. Serbyn, Z. Papić, and D. A. Abanin, Phys. Rev. B 90, 174302 (2014).

[7] M.Žnidarič, T. Prosen, and P. Prelovšek, Phys. Rev. B 77, 064426 (2008).

[8] J. H. Bardarson, F. Pollmann, and J. E. Moore, Phys. Rev. Lett. 109, 017202 (2012).

[9] M. Serbyn, Z. Papić, and D. A. Abanin, Phys. Rev. Lett. 110, 260601 (2013).

[10] F. Iemini, A. Russomanno, D. Rossini, A. Scardicchio, and R. Fazio, Phys. Rev. B 94, 214206 (2016).

[11] G. De Tomasi, S. Bera, J. H. Bardarson, and F. Pollmann, Phys. Rev. Lett. 118, 016804 (2017).

[12] B. Deissler, M. Zaccanti, G. Roati, C. D’Errico, M. Fattori, M. Modugno, G. Modugno, and M. Inguscio, Nat. Phys. 6, 354 (2010).

[13] D. Clément, A. F. Varòn, J. A. Retter, L. Sanchez-Palencia, A. Aspect, and P. Bouyer, New J. Phys. 8, 165 (2006).

[14] S. Krinner, D. Stadler, J. Meineke, J.-P. Brantut, and T. Esslinger, Phys. Rev. Lett. 115, 045302 (2015).

[15] S. S. Kondov, W. R. McGehee, W. Xu, and B. DeMarco, Phys. Rev. Lett. 114, 083002 (2015).

[16] M. Schreiber, S. S. Hodgman, P. Bordia, H. P. Luschen, M. H. Fischer, R. Vosk, E. Altman, U. Schneider, and I. Bloch, Science 349, 842 (2015).

[17] H. P. Lüschen, P. Bordia, S. Scherg, F. Alet, E. Altman, U. Schneider, and I. Bloch, Phys. Rev. Lett. 119, 260401 (2017).

[18] H. P. Lüschen, P. Bordia, S. S. Hodgman, M. Schreiber, S. Sarkar, A. J. Daley, M. H. Fischer, E. Altman, I. Bloch, and U. Schneider, Phys. Rev. X 7, 011034 (2017).

[19] U. Schollwöck, Rev. Mod. Phys. 77, 259 (2005).

[20] G. Xianlong, M. Polini, R. Asgari, and M. P. Tosi, Phys. Rev. A 73, 033609 (2006).

[21] S. H. Abedinpour, M. Polini, G. Xianlong, and M. P. Tosi, Phys. Rev. A 75, 015602 (2007).

[22] G. Xianlong and R. Asgari, Phys. Rev. A 77, 033604 (2008).

[23] S. Pilati, I. Zintchenko, M. Troyer, and F. Ancilotto [Eur. Phys. J. B (to be published)], arXiv:1801.02095.

[24] W. Li, G. Xianlong, C. Kollath, and M. Polini, Phys. Rev. B 78, 195109 (2008).

[25] M. A. L. Marques and E. K. U. Gross, Lect. Notes Phys. 620, 144 (2003).

[26] S. Das Sarma, S. He, and X. C. Xie, Phys. Rev. B 41, 5544 (1990).

[27] D. J. Boers, B. Goedeke, D. Hinrichs, and M. Holthaus, Phys. Rev. A 75, 063404 (2007).

[28] J. Biddle, B. Wang, D. J. Priour, and S. Das Sarma, Phys. Rev. A 80, 021603 (2009).
[29] S. Pilati and V. K. Varma, Phys. Rev. A 95, 013613 (2017).

[30] S. Aubry and G. André, Ann. Israel Phys. Soc. 3, 133 (1980).

[31] X. Li, X. Li, and S. Das Sarma, Phys. Rev. B 96, 085119 (2017).

[32] H. P. Luschen, S. Scherg, T. Kohlert, M. Schreiber, P. Bordia, X. Li, S. Das Sarma, and I. Bloch, arXiv:1709.03478.

[33] M. Olshanii, Phys. Rev. Lett. 81, 938 (1998).

[34] M. D. Girardeau, J. Math. Phys. 1, 516 (1960).

[35] M. D. Girardeau, Phys. Rev. A 82, 011607(R) (2010).

[36] L. Guan, S. Chen, Y. Wang, and Z. Q. Ma, Phys. Rev. Lett. 102, 160402 (2009).

[37] G. Zürn, F. Serwane, T. Lompe, A. N. Wenz, M. G. Ries, J. E. Bohn, and S. Jochim, Phys. Rev. Lett. 108, 075303 (2012).

[38] M. Modugno, New J. Phys. 11, 033023 (2009).

[39] S. Pilati, L. Barbiero, R. Fazio, and L. Dell'Anna, Phys. Rev. A 96, 021601(R) (2017).

[40] V. K. Varma, A. Lerose, F. Pietracaprina, J. Goold, and A. Scardicchio, J. Stat. Mech. (2017) 053101.

[41] P. N. Ma, S. Pilati, M. Troyer, and X. Dai, Nat. Phys. 8, 601 (2012).

[42] A. Bulgac, Y. L. Luo, P. Magierski, K. J. Roche, and Y. Yu, Science 332, 1288 (2011)

[43] A. Bulgac, M. McNeil Forbes, M. M. Kelley, K. J. Roche, and G. Wlazlowski, Phys. Rev. Lett. 112, 025301 (2014).

[44] F. Ancilotto, Phys. Rev. A 93, 053627 (2016).

[45] F. Ancilotto, Phys. Rev. A 92, 061602(R) (2015).

[46] I. Zintchenko, L. Wang, and M. Troyer, Eur. Phys. J. B 89, 180 (2016).

[47] D. M. Ceperley, J. Stat. Phys. 63, 1237 (1991).

[48] R. J. Magyar and K. Burke, Phys. Rev. A 70, 032508 (2004).

[49] J. P. Perdew and Y. Wang, Phys. Rev. B 45, 13244 (1992).

[50] P. Hoenberg and W. Kohn, Phys. Rev. 136, B864 (1964).

[51] G. Xianlong, M. Polini, D. Rainis, M. P. Tosi, and G. Vignale, Phys. Rev. Lett. 101, 206402 (2008).

[52] A. Bulgac, Annu. Rev. Nucl. Part. Sci. 63, 97 (2013).

[53] W. Kohn and L. J. Sham, Phys. Rev. 140, A1133 (1965).

[54] A. Ralston and H. S. Wilf, Mathematical Methods for Digital Computers (John Wiley and Sons, New York, 1960).

[55] W. H. Press, S. A. Teukolsky, W. T. Vetterling, and B. P. Flannery, Numerical Recipes (Cambridge University Press, Cambridge, England, 1992).

[56] With such choice the initial occupation of odd sites is between 1.8 and 2, while that of the even sites is between 0 and 0.15 . A more ordered initial state can be prepared by choosing a larger value for the well depth of the additional confining potential, e.g., three times the chosen value of $V_{0}$. However, we verified that the results of our time-dependent simulations are independent of the fine details of the initial state.

[57] B. Kramer and A. MacKinnon, Rep. Prog. Phys. 56, 1469 (1993).

[58] R. Mondaini and M. Rigol, Phys. Rev. A 92, 041601(R) (2015).

[59] P. Sierant and J. Zakrzewski, arXiv:1712.02628.

[60] S. Gopalakrishnan, K. Agarwal, E. A. Demler, D. A. Huse, and M. Knap, Phys. Rev. B 93, 134206 (2016).

[61] E. M. Stoudenmire and S. R. White, Annu. Rev. Condens. Matter Phys. 3, 111 (2012). 